\section{Características sociodemográficas, bienestar subjetivo y homofobia en una muestra de hombres gay en tres ciudades chilenas}

\author{
Socio-demographic characteristics, subjective \\ well-being, and homophobia experienced by \\ a sample of gay men from three cities in Chile \\ Características sociodemográficas, bem-estar \\ subjetivo e homofobia em uma amostra de \\ homens gays em três cidades chilenas
}

Jaime Barrientos-Delgado 1 Manuel Cárdenas-Castro 2 Fabiola Gómez-Ojeda ${ }^{1}$

\footnotetext{
1 Escuela de Psicología, Universidad Católica del Norte, Antofagasta, Chile. 2 Universidad de Valparaíso, Valparaíso, Chile.

\section{Correspondencia} F. Gómez-Ojeda Escuela de Psicología Universidad Católica del Norte.

Camino del Altar 03049, Lo Barnechea, Santiago / Región Metropolitana - 7700827 Chile.

fabigomez.o@gmail.com
}

\begin{abstract}
This article describes the socio-demographic characteristics of a sample of gay men in three cities in Chile, as well as experience with homophobia and subjective well-being. Snowball sampling was used to interview 325 gay men. The main findings included high levels of perceived discrimination and victimization, but interviewees reported higher levels of social well-being compared to studies elsewhere in the country. Age was related to differences in levels of social well-being, but not other variables. Individuals with university education reported higher levels of victimization and greater impact of discrimination on their lives. Gay men in Santiago reported a higher relative impact from incidents of aggression, but better levels of social well-being and happiness compared to those in other regions of Chile.
\end{abstract}

Male Homosexuality; Homophobia; Social Discrimination

\section{Resumen}

Este artículo describe y caracteriza sociodemográficamente a una muestra de hombres gay en tres ciudades de Chile. Además, describe sus niveles de homofobia y bienestar subjetivo. Mediante un muestreo en cadena tipo bola de nieve se encuestó a 325 hombres que se autodefinieron como gay. Entre los principales hallazgos se encontró altos niveles de discriminación y victimización percibida. Además en comparación con estudios similares efectuados en el país, los encuestados presentan niveles mayores de bienestar social. La edad de los encuestados aporta diferencias para los niveles de bienestar social y no para las otras medidas. También, las personas con estudios superiores reportan mayores niveles de victimización y un mayor impacto vital de las situaciones de discriminación. Asimismo, si bien las personas que residen en Santiago reportan un mayor impacto relativo de los hechos de agresión, presentan mejores niveles de bienestar social y felicidad en comparación a las personas que viven en otras regiones.

Homosexualidad Masculina; Homofobia; Discriminación Social 
Recientes mediciones realizadas por la Encuesta Mundial de Valores (WVS) y el Latinobarómetro aportan datos alentadores respecto a las actitudes de la sociedad chilena frente a la homosexualidad. Según estas fuentes, en 1990 un 76,9\% de los encuestados señalaba que la homosexualidad "nunca es justificable", porcentaje que ha disminuido a un 11,9\% en el año 2009 1. No obstante, otros estudios indican que, más bien, han emergido nuevas formas de manifestación del prejuicio hacia las minorías sexuales ${ }^{2}$, expresiones que serían más sutiles y menos explícitas. Los informes anuales realizados por agrupaciones sociales vinculadas a las minorías sexuales ratifican lo antes dicho 1,3 .

Un estudio reciente, realizado en el contexto de la Marcha del Orgullo LGBT chilena 4, indica que cerca del $80 \%$ de las personas lesbianas, gay, bisexuales y transexuales (LGBT) encuestadas, declaran haber sido víctimas de discriminación y agresión. Las burlas, los insultos y las amenazas son las situaciones más reportadas y los lugares más frecuentes: el barrio, ambientes religiosos y el lugar de estudios. Esta situación no es exclusiva de la sociedad chilena, ya que datos similares han sido reportados por encuestas realizadas en otros países de la región, como Argentina 5, Brasil ${ }^{6}$, Colombia 7 y México ${ }^{8}$.

Diversos países, incluido Chile, disponen de iniciativas orientadas al reconocimiento y protección de los derechos de las minorías sexuales (ej. ley anti-discriminación, regulación de las uniones civiles, matrimonio entre personas del mismo sexo o parentesco y adopción) 9, lo que ha generado un creciente interés por conocer las características demográficas y sociales de las personas pertenecientes a las minorías sexuales, transformándose, este conocimiento, en un tema de relevancia para la generación de políticas públicas en salud y educación en muchos países del mundo 10,11,12. En algunos países de América Latina existen estudios semejantes en esta población, focalizados en indagar aspectos de vulnerabilidad al VIH 13,14, sin embargo, en Chile aún no hay estudios que describan y caractericen a esta población. Son muy escasos los estudios existentes en América Latina sobre calidad de vida en esta población 15 .

\section{Efectos de la discriminación en la salud mental de población homosexual}

La homofobia, entendida como actitudes negativas hacia las minorías sexuales, aún existe en muchos países del mundo 9,16. Estas actitudes se traducen en violencia hacia las minorías sexuales. Un gran número de investigaciones ha reportado violencia y discriminación motivada por orientación sexual en contra de hombres gay, lesbianas y otras minorías sexuales, a saber: violencia física, acoso e intimidación, abuso verbal y asalto 12,17. En cuanto al trato discriminatorio, es frecuente que este se traduzca en marginación de espacios públicos y el rechazo por parte de personas cercanas dentro de la familia 18 o en las comunidades religiosas 19.

En relación al impacto que tiene la homofobia sobre la salud física y mental de las minorías sexuales, Meyer 20 propone que las personas que forman parte de grupos minoritarios (por ejemplo, hombres gay), prejuiciados socialmente, están expuestos a estresores sociales específicos que les afectan por su pertenencia a dichos grupos.

Por otro lado, diversos estudios han demostrado la existencia de una asociación entre la percepción de discriminación y haber sufrido malos tratos con diversos síntomas psicológicos tales como angustia y estrés $21,22,23$. Sin embargo, las experiencias derivadas de la violencia hacia las minorías sexuales no sólo se relacionan con sintomatología física y mental, sino que se relacionan también con una mayor vulnerabilidad al VIH y una mayor prevalencia de conductas de riesgo para la salud en esta población 23,24,25,26,27.

La mayor parte de la evidencia científica, respecto de los efectos de la violencia hacia gay y lesbianas, sobre la salud física y mental de las personas víctimas de ésta proviene de Estados Unidos o Europa y utilizan indicadores negativos de salud física y mental, indagando en la prevalencia de sintomatología física y mental 12,28. En América Latina existen pocos estudios sobre este tema, además, este enfoque patogénico deja fuera aspectos relevantes como el bienestar psicológico y social, así como el funcionamiento psicológico positivo o felicidad 29,30 .

Por ello, en Chile es relevante indagar en el posible impacto que la violencia ejercida hacia las minorías sexuales tiene sobre su salud física y mental, incorporando una mirada que ponga atención en indicadores positivos 12 .

Con el propósito de seguir avanzando en la generación de conocimiento contextualizado, respecto de las minorías sexuales en Chile y el eventual impacto que esto podría tener en su bienestar y calidad de vida, en este artículo reportamos las características sociodemográficas, los niveles de discriminación y agresión percibidos, así como los niveles de bienestar en una muestra de conveniencia de hombres gay en tres ciudades chilenas. 


\section{Método}

\section{Participantes}

La muestra final quedó conformada por 325 hombres que se auto-identificaron como gay. La edad fluctuó entre los 18 y los 64 años $(M=30,82$; $\mathrm{Mdn}=28$; DT = 9,81).

Las minorías sexuales son una población de difícil acceso ${ }^{15}$, por lo que no se cuenta con un marco muestral que permita hacer una selección aleatoria sobre esta población. Por ello, se utilizó un muestreo en cadena tipo "bola de nieve", tal como en estudios previos efectuados en la región 14. Los criterios de selección de la muestra fueron los siguientes: (a) que se autodefinieran como hombres gay, (b) tener una amplia red social, (c) lugar de residencia (Arica, Santiago o Valparaíso), y (d) ser mayor de 18 años.

\section{Análisis estadísticos}

Se efectuaron análisis descriptivos univariados y bivariados, considerando las variables edad, ciudad de residencia y nivel educativo como variables de segmentación. Para los análisis se utilizó el paquete estadístico SPSS (SPSS Inc., Chicago, Estados Unidos).

\section{Instrumento}

\section{- Medidas sociodemográficas}

a) Edad: se consulta en años cumplidos en el momento de ser encuestados. Con el propósito de facilitar el análisis de los datos, esta variable se recategorizó en tres tramos: 18-29, 30-44 y 45 o más años.

b) Ciudad de residencia: Santiago, Valparaíso o Arica.

c) Nivel de estudios más alto alcanzado: variable categórica con siete alternativas de respuesta que van desde educación básica incompleta hasta estudios de post-grado. A fin de facilitar el análisis de los datos y conformar grupos más homogéneos, esta variable se recategorizó en dos grupos: personas con estudios superiores (universitarios y/o técnicos superiores) y personas sin estudios superiores.

d) Nivel socioeconómico: se usó el indicador ESOMAR (European Society for Opinion and Marketing Research) 31, sistema de medición adaptado al contexto chileno ${ }^{32}$, el cual mide la posición económica y social de una familia en comparación con las demás, combinando en una matriz las variables nivel educativo y categoría ocupacional del principal sostenedor del hogar al que pertenece el encuestado. e) Religiosidad; se consulta a los participantes si se consideran religiosos (opciones de respuesta sí/no) y a qué religión adscriben.

\section{- Indicadores de homofobia}

a) Hechos de agresión 18: escala de 10 ítems que indaga en hechos de agresión vividos por gay y lesbianas y que son atribuidas a su orientación sexual. Incluye eventos de violencia física, verbal y psicológica con respuestas dicotómicas (sí/ no). Además, se consulta sobre la importancia atribuida a cada una de las situaciones vividas: ¿cuánto te ha impactado esta situación? Formato de respuesta Likert de seis opciones: 1 ("casi nada”) hasta 6 ("muchísimo"). Para esta aplicación se obtiene una consistencia interna elevada ( $\alpha=0.97$ ).

b) Hechos de discriminación 18: escala de 10 ítems que indaga en hechos de discriminación vividos por gay y lesbianas, los cuales son atribuidos directamente a su orientación sexual. Incluye haber sido objeto de discriminación en la familia, escuela, lugares públicos, entre otros (respuestas dicotómicas sí/no). Adicionalmente, se ha indagado en la importancia atribuida a cada una de las situaciones vividas: ¿ cuánto te ha impactado esta situación? Formato de respuesta Likert: 1 ("nada en absoluto") hasta 6 ("muchísimo"). Puntuaciones altas en esta escala indica altos niveles de discriminación. Se obtiene una consistencia interna elevada ( $\alpha=0.97$ ).

\section{- Medidas de bienestar subjetivo}

a) Escala de Felicidad Subjetiva 33, versión adaptada y validada para población nacional 34 . La escala está compuesta por 4 ítems con respuesta Likert, cuya corrección se hace mediante la sumatoria de los puntajes obtenidos y se dividen en el número total de ítem. Las opciones de respuesta van de 1 hasta 6 . Se obtuvo un índice de consistencia interna de $\alpha=0.65$.

b) Escala de Satisfacción con la Vida 35 . Se utiliza versión adaptada para población nacional 36 . Esta versión está compuesta de 5 ítems, con opciones de respuesta tipo Likert que van de 1 ("totalmente en desacuerdo") hasta 6 ("totalmente de acuerdo”). Para esta aplicación se obtiene un alpha de Cronbach de 0.89 .

c) Bienestar Social 37. Versión en español de Blanco \& Díaz 38 y validada en población nacional 39 , escala compuesta por 33 ítems, distribuidos en 5 dimensiones: Integración Social, Aceptación Social, Contribución Social, Actualización Social y Coherencia Social. Las opciones de respuesta Likert van desde 1 ("totalmente en desacuerdo") hasta 6 ("totalmente de acuerdo"). Puntuaciones 
altas indican buen ajuste social. Para esta aplicación el coeficiente alpha de Cronbach fue de 0.89 . La fiabilidad para las dimensiones van de $\alpha=0.38$ (Contribución Social) a $\alpha=0.86$ (Coherencia Social).

\section{Procedimiento}

El presente estudio forma parte de una investigación más amplia que se proponía, por un lado, evaluar el estigma y la discriminación existente hacia hombres gay en Chile, así como la relación que se establece entre este estigma y cuestiones como el VIH y la orientación sexual y también, el efecto del estigma en la calidad de vida y el bienestar. En función de estos objetivos, los datos fueron recolectados en las ciudades de Santiago, Arica y Valparaíso (criterio de selección), ya que comprenden las ciudades que presentan una alta prevalencia de VIH/SIDA en el país 40 . Asimismo, se escogió dichas ciudades, ya que en ellas hay organizaciones LGBT que facilitarían el acceso a dicha población y, por tanto, la realización del estudio. Es preciso indicar que en este artículo sólo se reportan una parte de todos los resultados encontrados.

Para hacer el estudio, en cada región, se seleccionó un coordinador de terreno, y se capacitó un grupo de encuestadores. El instrumento fue aplicado cara a cara. Los participantes fueron informados de los objetivos del estudio y firmaron una carta de consentimiento informado en la que se aseguraba la confidencialidad de los datos entregados para esta investigación. Este estudio recibió la aprobación del comité de ética de la Universidad y del Fondo Nacional de Desarrollo Científico y Tecnológico (FONDECYT).

Para seleccionar la muestra se escogieron las "semillas" según los criterios de selección previamente establecidos. Se seleccionaron tres "semillas" en cada ciudad, en base a la información entregada previamente por informantes clave. Estas "semillas" sirvieron de punto de partida del reclutamiento de la muestra. Después de completar la encuesta, cada semilla debía invitar a otros tres hombres gay, quienes debían cumplir los criterios de elegibilidad para participar en el estudio. Estos nuevos participantes eran contactados por el equipo de investigación para su inclusión en la muestra. Este proceso se repitió hasta que las nuevas semillas no eran capaces de reclutar a nuevos participantes y una vez sucedía esto, se concluía la recogida de datos en la ciudad.

\section{Resultados}

\section{Descripción de los participantes de la muestra}

En la Tabla 1 puede apreciarse la distribución de los participantes según variables socio-demográficas.

Es una muestra joven, que se concentra entre los 18 y 29 años (52,9\%), en la que sólo un 12,9\% de los encuestados tiene más de 45 años. Es una población educada, con niveles de estudio que se agrupan en dos categorías principales: "educación media completa o técnica incompleta" $(29,8 \%)$ y "educación universitaria incompleta o técnica completa" $(38,2 \%)$ y cuyo nivel socioeconómico es medio y medio alto $(68,4 \%)$.

Casi la mitad de los encuestados reside en la ciudad de Santiago, es decir, en la capital del país (49,5\%), mientras que el resto se distribuye de forma casi homogénea en las otras dos ciudades: Valparaíso $(24,6 \%)$ y Arica (24\%). Además, la mayor parte de los encuestados no se considera una persona religiosa $(63,9 \%)$ y aquellos que sí lo hacen $(36,1 \%)$ adscriben, mayoritariamente, a la religión Católica (78\%). Respecto de su posicionamiento político, la mayor parte de los participantes opta por posicionar sus ideas en torno al centro político $(63,4 \%)$.

\section{Medidas de homofobia}

Tal como se puede apreciar en la Tabla 2 los hechos de agresión más reportados son los que refieren a violencia verbal, más específicamente, burlas e insultos. Las amenazas también constituyen un hecho de agresión reportado por uno de cada cuatro encuestados. Una proporción igualmente importante de los encuestados, más del $20 \%$, reporta haber sufrido asaltos o robos sin violencia, por el hecho de ser identificado como gay.

Ante la pregunta por el grado en que dichas acciones impactaron en su vida, las personas asignan niveles de importancia relativa a dichos actos que resultan interesantes de analizar. En la Tabla 2 pueden apreciarse las puntuaciones medias para el grado de impacto que éstas han tenido en la vida de las personas encuestadas. Resalta que la agresión física $(M=3,77)$ y las burlas $(M=3,5)$ resultan los hechos de mayor impacto vital, y al mismo tiempo las burlas son los hechos de mayor ocurrencia.

$\mathrm{Si}$ analizamos diferencias, según grupos de edad, no se observan diferencias estadísticamente significativas para hechos de agresión, ni para la importancia relativa atribuida a éstos. 
Caracterización sociodemográfica de los encuestados.

\begin{tabular}{|c|c|c|}
\hline Variables & $\%$ & $n$ * \\
\hline \multicolumn{3}{|l|}{ Edad (años) } \\
\hline $18-29$ & 52,9 & 172 \\
\hline $30-44$ & 34,2 & 111 \\
\hline 45 o más & 12,9 & 42 \\
\hline \multicolumn{3}{|l|}{ Nivel socioeconómico } \\
\hline Medio bajo & 14,4 & 47 \\
\hline Medio & 32,9 & 107 \\
\hline Medio alto & 30,5 & 99 \\
\hline Alto & 14,8 & 48 \\
\hline NS/NR & 7,4 & 24 \\
\hline \multicolumn{3}{|l|}{ Ciudad } \\
\hline Santiago & 49,6 & 161 \\
\hline Valparaíso & 24,6 & 80 \\
\hline Arica & 24,0 & 78 \\
\hline NR & 1,8 & 6 \\
\hline \multicolumn{3}{|l|}{ Nivel educativo } \\
\hline Media incompleta o menos estudio & 6,2 & 20 \\
\hline Media completa o Técnica incompleta & 29,8 & 97 \\
\hline Universitaria incompleta o Técnica completa & 38,2 & 124 \\
\hline Universitaria completa y/o postgrado & 24,9 & 81 \\
\hline NS/NR & 0,9 & 3 \\
\hline \multicolumn{3}{|l|}{ Religión } \\
\hline Sí & 35,4 & 115 \\
\hline No & 62,8 & 204 \\
\hline NS/NR & 1,8 & 6 \\
\hline \multicolumn{3}{|l|}{ Tipo de Religión } \\
\hline Católica & 73,9 & 85 \\
\hline Evangélica & 7,8 & 9 \\
\hline Otra & 11,3 & 13 \\
\hline NS/NR & 7,0 & 8 \\
\hline \multicolumn{3}{|l|}{ Posicionamiento político ** } \\
\hline Derecha & 9,5 & 31 \\
\hline Centro & 63,4 & 206 \\
\hline Izquierda & 25,3 & 82 \\
\hline NS/NR & 1,8 & 6 \\
\hline
\end{tabular}

NR: no responde; NS: no sabe.

* Número de sujetos que responde;

** Esta variable fue recodificada para obtener los tres grupos reportados.

En cambio, sí se observan diferencias estadísticamente significativas según ciudad de residencia $\left(F_{(2,314)}=5,6 ; p=0,004 ; \eta_{p}{ }^{2}=0,20\right)$. Las personas que residen en Arica reportan mayores niveles de agresión $(M=7,78)$, en comparación a las personas que viven en Santiago $(M=6,75)$. A pesar de estos resultados, el impacto vital de las agresiones es mayor en las personas que viven en Santiago $(M=3,79)$, en comparación a las que lo hacen en Arica $(M=2,87)$ y esta diferencia es estadísticamente significativa $\left(\mathrm{F}_{(2,258)}=10,10\right.$; $\left.\mathrm{p}=0,000 ; \eta_{\mathrm{p}}{ }^{2}=0,26\right)$. No se observan diferencias estadísticamente significativas entre las otras ciudades para hechos de agresión reportados.

Los análisis por nivel de estudios alcanzados indican diferencias estadísticamente significativas en los niveles de agresión $\left(\mathrm{t}_{(318)}=3,79 ; \mathrm{p}=\right.$ 0,$000 ; d=0,42$ ). Las personas con estudios su- 
Tabla 2

Acciones de agresión motivada por la orientación sexual y su impacto vital.

\begin{tabular}{lll}
\hline Acciones de agresión & $\% *$ & $\mathbf{M} * *$ \\
\hline Burlas & 73,2 & 3,50 \\
Insultos & 55,8 & 3,47 \\
Amenazas & 26,7 & 3,29 \\
Intento de agresión física & 21,6 & 3,48 \\
Agresión física & 16,6 & 3,77 \\
Intento de agresión sexual & 14,7 & 3,11 \\
Agresión sexual & 11,5 & 3,28 \\
Chantaje & 18,9 & 3,39 \\
Asalto o robo sin violencia & 22,4 & 3,28 \\
Asalto o robo con violencia & 13,7 & 3,29 \\
\hline
\end{tabular}

* Frecuencia con la que se ha vivido las acciones de agresión señaladas;

** Media del impacto vital que cada hecho de agresión ha tenido sobre la vida de las personas que los han vivenciado (escala que va de 1 "casi nada" hasta 6 "muchísimo).

periores presentan niveles más altos de agresión ( $\mathrm{M}=7,61)$, en comparación con las personas que no tienen estudios superiores $(M=6,48)$. No se encuentran diferencias estadísticamente significativas para el impacto vital.

En la Tabla 3 podemos revisar el reporte de actos de discriminación a los que han sido sometidos los participantes, debido a su orientación sexual. También, se ha incorporado la media del impacto vital atribuido por los participantes a dicha acción de discriminación.

Los hechos de discriminación más reportados son aquellos asociados al hostigamiento perpetrado por vecinos, funcionarios públicos y no haber sido contratado o haber sido despedido de un trabajo. No obstante, los hechos que más han impactado la vida de los encuestados refieren a aquellos vividos en el ámbito familiar $(\mathrm{M}=4,01)$ y los hechos de discriminación asociados al trabajo ( $\mathrm{M}=3,61)$.

$\mathrm{Al}$ analizar si existen diferencias en los hechos de discriminación entre los diferentes grupos de edad no se observan diferencias estadísticamente significativas.

Las personas que viven en Santiago reportan menos niveles de discriminación $(\mathrm{M}=7,39)$ en comparación a los encuestados que viven en Arica y Valparaíso ( $\mathrm{M}=9,00$ y $\mathrm{M}=8,54$, respectivamente) y estas diferencias son estadísticamente significativas $\left(\mathrm{F}_{(2,315)}=14,29 ; \mathrm{p}=0,004\right.$; $\left.\eta_{\mathrm{p}}{ }^{2}=0,35\right)$. Sin embargo, las personas que viven en Santiago reportan un mayor impacto vital de estos hechos $(M=3,73)$ en comparación a las que viven en Arica $(M=3,14)$ y Valparaíso ( $M=3,17)$, y esta diferencia es estadísticamente significativa $\left(\mathrm{F}_{(2,202)}=4,13 ; \mathrm{p}=0,02 ; \eta_{\mathrm{p}}{ }^{2}=0,20\right)$.

Por otra parte, las personas con estudios superiores presentan niveles mayores de discriminación $(\mathrm{M}=8,64)$, en comparación a las personas que no los tienen $(M=7,07)$. Estas diferencias son significativas estadísticamente $\left(\mathrm{t}_{(319)}=\right.$ 5,$77 ; \mathrm{p}=0,000 ; \mathrm{d}=0,60$ ).

\section{Medidas de bienestar}

La media para la medida de felicidad fue de 4,19 (DT $=1,01)$. La media de satisfacción vital fue de 4,14 (DT = 1,31). En tanto, para la medida de bienestar social se obtienen medias que van entre 2,93 a 4,16. En las subdimensiones integración social $(M=4,16)$, en aceptación social $(M=3,59)$, contribución social $(\mathrm{M}=3,74)$, actualización social $(M=3,46)$ y coherencia social $(M=2,93)$.

Se observan diferencias estadísticamente significativas en los niveles reportados de felicidad entre los encuestados que viven en Santiago $(\mathrm{M}=4,37)$ y los residentes de Valparaíso $(\mathrm{M}=$ 3,87 ), siendo estos últimos los que obtienen niveles significativamente menores $\left(\mathrm{F}_{(2,315)}=7,13\right.$; $\left.p=0,001 ; \eta_{p}^{2}=0,22\right)$. Sin embargo, no se observan diferencias significativas en los niveles de felicidad según tramos de edad y nivel educativo. Tampoco, se observan diferencias significativas en los niveles de satisfacción según edad, ciudad y nivel educativo.

En bienestar social se observan diferencias estadísticamente significativas según edad $\left(\mathrm{F}_{(2,322)}=4,74 ; \mathrm{p}=0,005 ; \eta_{\mathrm{p}}{ }^{2}=0,13\right)$. Las personas de 45 años o más presentan niveles menores de bienestar social $(M=3,29)$, en comparación a las que tienen entre 18 y 29 años de edad $(\mathrm{M}=$ $3,68)$. En cuanto a las diferencias según ciudad, las personas que viven en Santiago $(\mathrm{M}=3,73)$ reportan mayores niveles de bienestar social que aquellos que viven en Arica $(\mathrm{M}=3,50)$ y Valparaíso $(M=3,47)$, y estas diferencias son estadísticamente significativas $\left(\mathrm{F}_{(2,316)}=4,38 ; \mathrm{p}=0,013\right.$; $\left.\eta_{\mathrm{p}}{ }^{2}=0,18\right)$.

Finalmente, se encuentran diferencias En los niveles de bienestar social entre las personas que tienen estudios superiores $(M=3,41)$ y las que no los tienen $(\mathrm{M}=3,90)$, siendo las primeras las que reportan menor bienestar social $\left(\mathrm{t}_{(320)}=5,84 ; \mathrm{p}=\right.$ 0,000; $d=0,64)$.

\section{Discusión}

Este artículo es un primer acercamiento a la descripción y caracterización de la población gay 
Acciones de discriminación motivada por la orientación sexual y su impacto vital.

\begin{tabular}{lcc}
\hline Acciones de discriminación & $\%$ * & M ** \\
\hline No haber sido contratado o despedido del trabajo & 22,3 & 3,61 \\
Prohibir entrada o permanencia en un local & 14,2 & 2,87 \\
Mal atendido por funcionarios públicos & 25,8 & 3,51 \\
No aceptado o expulsado de lugar estudios & 9,3 & 2,91 \\
No aceptado o expulsado de grupo de amigos & 19,8 & 3,51 \\
Molestado u hostigado por vecinos & 32,5 & 3,13 \\
No aceptado o expulsado de agrupación social & 10,6 & 3,21 \\
No aceptado o expulsado de ambiente familiar & 18,0 & 4,01 \\
No aceptado o expulsado de ambiente religioso & 16,1 & 3,38 \\
Maltrato verbal o físico o negación de ayuda de la policía & 19,3 & 3,59 \\
\hline
\end{tabular}

* Frecuencia con la que se ha vivido las acciones de discriminación señaladas;

** Media del impacto vital que cada hecho de discriminación ha tenido sobre la vida de las personas que los han vivenciado (escala que va de 1 "casi nada" hasta 6 "muchísimo).

chilena. La mayoría de los estudios previos han estado orientados a indagar exclusivamente en la homofobia $2,41,42$ y sus efectos negativos sobre la salud física y mental, utilizando enfoques que no incluyen el bienestar y la felicidad 22,25. Por tanto, este estudio es inédito, ya que abre una línea de trabajo que puede complementar los estudios existentes.

Además, conocer algunas características sociodemográficas de este grupo podría contribuir a la orientación de políticas públicas y ser línea base para futuros estudios de salud pública en minorías sexuales 43 . Sin embargo, se debe tener presente que los resultados obtenidos, más bien representan a un sector de la población estudiada que, por sus características, se sitúa en un contexto de protección frente al prejuicio y sus posibles efectos. Se trata de personas jóvenes, con altos niveles educacionales, pertenecientes a niveles socioeconómico medio a alto y, si bien la muestra corresponde a tres ciudades chilenas, en su mayoría son personas que viven en la capital de nuestro país, con los beneficios propios de vivir en una zona metropolitana (buen acceso a prestaciones de salud, mayor visibilidad de organizaciones sociales de apoyo, mejores y más espacios de sociabilidad, entre otros) 11.

No obstante, al realizar análisis más detallados, encontramos que son las personas con estudios superiores las que reportan mayores niveles de agresión y un mayor impacto vital de las situaciones de discriminación. Es posible que las personas con mayores niveles de estudio desarrollen una sensibilidad mayor hacia el reconocimiento de situaciones de violencia psicológica y física, así como mayor conciencia de sus derechos. Futuros estudios deberán profundizar en esto.

Por otro lado, las personas que residen en Santiago, si bien reportan un mayor impacto relativo de los hechos de agresión vividos, presentan mejores niveles de bienestar social y felicidad en comparación a las personas que viven en otras regiones. Estos resultados confirman que vivir en una ciudad grande podría convertirse en un factor protector 11 , toda vez que brinda a las personas mejores redes de soporte social que les permitan sortear de mejor forma los efectos negativos de la homofobia. Se ha visto que muchas personas pertenecientes a minorías sexuales deben abandonar sus lugares de origen y se desplazan a zonas urbanas buscando redes sociales de apoyo y modelos de vida gay o lésbicos positivos. Esto les permite constatar que las personas pertenecientes a grupos estigmatizados socialmente, pueden tener éxito en sus relaciones íntimas y lograr espacios de satisfacción vital 44.

Por otro lado, diversos estudios han mostrado cómo la población gay y lésbica difiere, según la edad, en diversas variables tales como violencia y salud 11,21,45. En relación a ello, es relevante destacar que en este estudio, a diferencia de lo reportado por Kertzner et al. 46, las personas más jóvenes son las que reportan niveles mayores de bienestar social. Además, la edad de los encuestados no es significativa para explicar diferencias en discriminación y agresión, a diferencia de lo que ha sido reportado en otros contextos 11.

En comparación con la población general chilena, los participantes de este estudio presentan menores niveles de felicidad ${ }^{34}$. Sin embargo, 
en comparación a estudios similares efectuados en el país, los encuestados presentan niveles mayores de bienestar social 29. Estos resultados son importantes, ya que se ha reportado que algunas personas pertenecientes a minorías sexuales presentan altos niveles de resiliencia y mecanismos de defensa eficaces para hacer frente a la estigmatización de la que son objeto y pueden dar un significado positivo a las experiencias de discriminación vividas 47 . Otros estudios reportan una asociación entre variables como apoyo social y sentido de pertenencia a una comunidad LGBT y buenos niveles de bienestar y calidad de vida 48 . Es posible que, dado las características de la población estudiada, estas sean variables que expliquen dichos niveles de bienestar. Se sugiere incluirlas en futuros estudios.

Coherentemente con lo reportado por estudios previos 18,29, los resultados de esta investigación demuestran la persistencia de la discriminación y agresión percibida. Los datos recabados indican el impacto diferencial que ha tenido cada acción de discriminación vivida, independientemente, de la magnitud de su ocurrencia. Se constata que, si bien las situaciones con mayor ocurrencia corresponden al ámbito público, aquellas que más impactan la vida de las personas encuestadas se sitúan en el ámbito privado (rechazo de su ambiente familiar). En este sentido, la familia lejos de ser un espacio de protección, contención y refuerzo de la autoestima, se convierte en una fuente de estrés proximal 49 .

Los resultados refuerzan la necesidad de incorporar en la comprensión de la homofobia y sus efectos la visión de la población víctima de ésta. En Chile poco se ha avanzado el respecto. Gómez \& Barrientos 29 han señalado que las experiencias de agresión, debidas a la orientación sexual, tienen un efecto negativo sobre el bienestar psicológico y social, y tienen relación con la aparición de sintomatología depresiva. De allí que sea necesario, incorporar en futuras investigaciones análisis estadísticos que permitan corroborar estas asociaciones y avanzar en la propuesta de nuevos modelos más comprensivos de la situación de vulnerabilidad de las personas que pertenecen a minorías sexuales.

Es necesario tener presente, que durante los últimos dos años en Chile, se ha abierto el debate respecto de los derechos de las minorías sexuales y se ha generado en la opinión pública una creciente sensibilidad frente al tema, principalmente, impulsada por el terrible asesinato de Daniel Zamudio, joven que fue brutalmente asesinado debido a su homosexualidad. Como reacción frente a este crimen, la clase política chilena promulgó una ley anti-discriminación, ley que las organizaciones sociales venían exigiendo hace décadas. Este contexto social podría explicar, en parte, los resultados obtenidos, dado que la percepción de vulnerabilidad por orientación sexual dependerá de cuán saliente se hagan, tanto la homofobia como los derechos ciudadanos de las minorías sexuales.

Especialmente relevante es indagar, en futuras investigaciones, sobre las inequidades de salud a las que se ve enfrentada la población LGBT 50 , ya que se ha reportado que las minorías sexuales enfrentan diversas barreras que impiden un acceso equitativo a servicios de salud, lo que podría tener un gran impacto en su calidad de vida 11,44,51. Por ejemplo, el miedo de ser dicriminados por los proveedores de salud que trabajan en los centros, así como la insatisfacción con los servicios de salud, podrían limitar el acceso de la población LGBT a los servicios de salud.

Además, en futuros estudios, se sugiere incorporar a población heterosexual como grupo control, ya que, de este modo, podemos comparar a la población gay con la heterosexual y llegar a conclusiones más precisas respecto del efecto diferencial de la discriminación y agresión sobre las minorías sexuales, así como de las diferencias en indicadores de salud y bienestar subjetivo existentes en ambos grupos, tal como lo proponen otros estudios 52,53 .

En cuanto a las limitaciones de este estudio existen diversos aspectos que deben ser considerados. El muestreo en poblaciones de difícil acceso es un desafío enorme para la investigación social. Este estudio ha intentado aproximarse a esta población usando un muestreo en cadena de tipo bola de nieve, el cual comienza con la incorporación de "semillas" (personas que poseen una amplia red de contactos) que nos permiten un ingreso menos intrusivo en la población objetivo. Sin embargo, este tipo de muestreo también tiene sus limitaciones, ya que se ha señalado que no permite una selección aleatoria de los participantes, lo que dificultaría la posibilidad de generalización de los hallazgos al conjunto de la población gay chilena. De este modo, este estudio recluta una muestra de conveniencia de hombres gay de tres ciudades del país y no representa a toda la población gay chilena, aún cuando se buscó obtener una muestra heterogénea. De lo anterior se deduce la importancia que tiene en futuros estudios incorporar nuevos métodos de muestreo tales como el muestreo conducido por el encuestado 54,55 o aquellos basados en los lugares y horarios de reunión de los miembros del grupo 56,57. Asimismo, futuros estudios deberían usar otras dimensiones para escoger las ciudades a estudiar (diferentes a la prevalencia del VIH), considerando que, si bien el VIH afecta prepon- 
derantemente a hombres que tienen sexo con otros hombres (HSH) (entre los que se incluyen a los hombres gay), hay muchos hombres que tienen sexo con otros hombres que no se identifican como gay.

También, futuros estudios deberían seguir profundizando en la recogida de datos sociodemográficos básicos sobre la población LGBT. Igualmente, futuros estudios deberían incluir nuevas medidas sociodemográficas como aquellas relativas a las diversas modalidades de constitución de pareja y familia en parejas del mismo sexo, ya que orientarían la discusión sobre nuevos modos de conyugalidad, pero también sobre los nuevos modos de constitución familiar en nuestro país.

Por último, futuros estudios deberían profundizar en una mirada del curso de la vida de temas como la violencia y la salud en población LGBT. La influencia de las diferencias de edad y generacionales en las vivencias de prejuicio y sus efectos en la salud de la población LGBT deben ser reconocidas. Estudios de tipo longitudinal deberían ser efectuados para comprender mejor el efecto de las diferencias de edad y generacionales en salud y violencia ${ }^{11}$.

\section{Resumo}

Este artigo descreve e caracteriza sociodemograficamente uma amostra de homens gay em três cidades do Chile, abordando também seus niveis de homofobia e bem-estar subjetivo. Por meio de uma amostra em série do tipo bola de neve foram entrevistados 325 homens autoidentificados como gay. Entre os principais achados evidenciaram-se altos níveis de discriminação e vitimização percebida, em comparação com estudos similares realizados no país. Os entrevistados apresentaram níveis maiores de bem-estar social. A idade dos entrevistados aporta diferenças para os níveis de bem- estar social e não para as outras medidas. As pessoas com estudos superiores reportam também maiores níveis de vitimização e um maior impacto vital das situações de discriminação. Adicionalmente, embora as pessoas que residem em Santiago reportem um maior impacto de agressões, eles apresentam melhores nivveis de bem-estar social e felicidade em comparação com pessoas que moram em outras regiões.

Homossexualidade Masculina; Homofobia;

Discriminação Social

\section{Colaboradores}

J. Barrientos-Delgado coordinó tanto la investigación que dio lugar al artículo, como el trabajo de elaboración de éste. Además participó activamente en su construcción. M. Cárdenas-Castro participó en la coordinación de la investigación y es el coordinador metodológico. Además, participó activamente en la construcción del artículo. F. Gómez-Ojeda participó en el proceso de análisis de datos y en la construcción del artículo.

\section{Agradecimientos}

Este artículo contó con el apoyo de proyecto FONDECYT 1110423, titulado Homofobia y Efectos Psicosociales en la Calidad de Vida de Homosexuales en Chile: Hacia un Modelo Comprensivo. 


\section{Referencias}

1. Movimiento de Integración y Liberación Homosexual. XI informe anual de derechos humanos de la diversidad sexual en Chile. http://www.movilh. cl (accedido el 03/Mar/2013).

2. Cárdenas M, Barrientos J. Actitudes explícitas e implícitas hacia los hombres homosexuales en una muestra de estudiantes universitarios en Chile. Psykhe (Santiago) 2008; 17:17-25.

3. Movimiento de Integración y Liberación Homosexual. X informe anual de derechos humanos de la diversidad sexual en Chile. http://www.movilh. cl (accedido el 10/Oct/2012).

4. Barrientos J, Díaz J, Muñoz F. Derechos, política y diversidad sexual. Segunda encuesta marcha por la diversidad sexual, Santiago de Chile 2011. Santiago: Universidad Católica del Norte/Movimiento por la Diversidad Sexual; 2012.

5. Jones D, Libson M, Hiller R. Sexualidad, política y violencia. La marcha del orgullo GLTTBI Buenos Aires 2005, segunda encuesta. Buenos Aires: Editorial Antropofagia; 2006.

6. Carrara S, Ramos S, Lacerda P, Medrado B, Vieira N. Política, dereitos, violencia e homossexualidade. Pesquisa 5a Parada da Diversidade - Pernambuco 2006. Rio de Janeiro: Centro Latino-Americano em Sexualidade e Direitos Humanos, Instituto de Medicina Social, Universidade do Estado do Rio de Janeiro; 2007.

7. Brigeiro M, Castillo E, Murad R. Encuesta LGBT: sexualidad y derechos. Participantes de la Marcha de la Ciudadanía. Río de Janeiro: Centro LatinoAmericano em Sexualidade e Direitos Humanos, Instituto de Medicina Social, Universidade do Estado do Rio de Janeiro; 2009.

8. Brito A, Jiménez de Sandi A, Sívori H, Lacerda P, Glockner N, De La Garza L. Política, derechos, violencia y sexualidad. Encuesta de la Marcha del Orgullo y la Diversidad Sexual de la Ciudad de México - 2008. Rio de Janeiro: Centro de Estudos e Pesquisa em Saúde Coletiva, Instituto de Medicina Social, Universidade do Estado do Rio de Janeiro; 2012.

9. Cáceres CF, Pecheny M, Frasca T, Raupp R, Pocahy F. Review of legal frameworks and the situation of human rights related to sexual diversity in low and middle income countries. http://www.sxpolitics. org/wp-content/uploads/2009/03/review-of-le gal-frameworks.pdf (accedido el 20/Nov/2012).

10. Herek GM, Norton AT, Allen TJ, Sims CL. Demographic, psychological, and social characteristics of self-identified lesbian, gay, and bisexual adults in a US probability sample. Sex Res Social Policy 2010; 7:176-200.

11. Institute of Medicine. The health of lesbian, gay, bisexual, and transgender people: building a foundation for better understanding. Washington DC: The National Academies Press; 2011.

12. Mulé N, Rossi L, Deeprose B, Jackson B, Daley A, Travers A, et al. Promoting LGBT health and wellbeing through inclusive policy development. Int J Equity Health 2009; 8:1-11.
13. Perez-Brumer AG, Konda KA, Salvatierra HJ, Segura ER, Hall ER, Montano SM, et al. Prevalence of HIV, STIs, and risk behaviors in a cross-sectional community- and clinic-based sample of men who have sex with men (MSM) in Lima, Peru. PLoS One 2013; 8:1-5.

14. Paz-Bailey G, Miller W, Shiraishi RW, Jacobson JO, Abimbola TO, Chen SY. Reaching men who have sex with men: a comparison of respondent-driven sampling and time-location sampling in Guatemala City. AIDS Behav 2013; 17:3081-90.

15. Ghorayeb DB, Dalgalarrondo P. Homosexuality: mental health and quality of life in a Brazilian socio-cultural context. Int J Soc Psychiatry 2011; 57:496-500.

16. Harding R. Regulating sexuality: Legal consciousness in lesbian and gay lives. Oxford: Routledge; 2011.

17. King M, McKeown E, Warner J, Ramsay A, Johnson $\mathrm{K}$, Cort C, et al. Mental health and quality of life gay men and lesbians in England and Wales. $\mathrm{Br} \mathrm{J}$ Psychiatr 2003; 183:552-8.

18. Barrientos J, Silva J, Catalán S, Gómez F, Longueira J. Discrimination and victimization: parade for lesbian, gay, bisexual, and transgender (LGBT) pride, in Chile. J Homosex 2010; 57:760-75.

19. Programa Conjunto de las Naciones Unidas sobre el VIH/SIDA; Organización de las Naciones Unidas para la Educación, la Ciencia y la Cultura; Ministerio de Salud; Vivo Positivo; Asosida. Índice compuesto de estigma y discriminación hacia hombres homosexuales, otros HSH y mujeres transexuales en Chile (ICED): síntesis de estudio. Santiago: Programa Conjunto de las Naciones Unidas sobre el VIH/SIDA; Organización de las Naciones Unidas para la Educación, la Ciencia y la Cultura; Ministerio de Salud; Vivo Positivo; Asosida; 2012.

20. Meyer I. Prejudice, social stress, and mental health in lesbian, gay, and bisexual populations: conceptual issues and research evidence. Psychol Bull 2003; 129:674-97.

21. Huebner D, Rebchook G, Kegeles S. Experiences of harassment, discrimination, and physical violence young gay and bisexual men. Am J Public Health 2004; 94:1200-3.

22. Mays V, Cochran S. Mental health correlates of perceived discrimination among lesbian, gay, and bisexual adults in the Unites States. Am J Public Health 2001; 91:1869-76.

23. Barrientos J, Vidal F, Cárdenas M. Homophobia and quality of life among people living with HIV in Chile. Int J Hisp Psychol 2012; 5:195-205.

24. Hamilton CJ, Mahalik JR. Minority stress, masculinity, and social norms predicting gay men's health risk behaviors. J Couns Psychol 2009; 1:132-41.

25. Hatzenbuehler ML, Nolen-Hoeksema S, Erickson SJ. Minority stress predictors of HIV risk behavior; substance use, and depressive symptoms: results from a prospective study of bereaved gay men. Health Psychol 2008; 27:455-62. 
26. Jarama SL, Kennamer JD, Poppen P, Hendricks M, Bradford J. Psychosocial, behavioral, and cultural predictors of sexual risk for HIV infection among latino men who have sex with men. AIDS Behav 2005; 9:513-23.

27. Pascoe E, Richman L. Perceived discrimination and health: a meta-analytic review. Psychol Bull 2009; 135:531-54.

28. Wong C, Tang C. Personality, psychosocial variables, and life satisfaction of Chinese gay men in Hong Kong. J Happiness Stud 2003; 4:285-95.

29. Gómez F, Barrientos J. Efectos del prejuicio sexual en la salud mental de gays y lesbianas, en la ciudad de Antofagasta, Chile. Sex Salud Soc (Rio J) 2012; 10:100-23.

30. Malta M, Magnanini MMF, Mello MB, Pascom ARP, Linhares Y, Bastos F. HIV prevalence among female sex workers, drug users and men who have sex with men in Brazil: a systematic review and metaanalysis. BMC Public Health 2010; 10:1-16.

31. Reif K, Marbeau Y, Quatresooz J, Vancraeynest D. Harmonization of demographics. progress report of the ESOMAR working party. In: ESOMAR Congress. Luxembourg City: European Society for Opinion and Marketing Research; 1991. p. 1-41.

32. Adimark. El nivel socio económico ESOMAR. Manual de aplicación. http://www.microweb.cl/ idm/documentos/ESOMAR.pdf (accedido el 10/ Oct/2007).

33. Lyubomirsky S, Lepper H. A measure of subjective happiness: preliminary reliability and construct validation. Soc Indic Res 1999; 46:137-55.

34. Vera P, Celis K, Córdova N. Evaluación de la felicidad: análisis psicométrico de la Escala de Felicidad Subjetiva en población chilena. Ter Psicol 2011; 29:127-33.

35. Diener E, Emmons RA, Larsen RJ, Griffin S. The satisfaction with life scale. J Pers Assess 1985; 49:71-5.

36. Cárdenas M, Barrientos J, Bilbao M, Páez D, Gómez F, Asún D. Estructura factorial de la escala de satisfacción con la vida en una muestra de estudiantes universitarios chilenos. Rev Mex Psicol (1984) 2012; 29:157-64.

37. Keyes C. Social well-being. Soc Psychol Q 1998; 61:121-40.

38. Blanco A, Díaz D. El bienestar social: su concepto y medición. Psicothema 2005; 17:582-9.

39. Cárdenas M, Barrientos J, Bilbao M. Adaptación y validación chilena de la escala de Bienestar Social (Social Well-Being Scale). Antofagasta: Escuela de Psicología, Universidad Católica del Norte; s.f.

40. Ministerio de Salud. Circular B/51 no 35 de 14 octubre 2010. Vigilancia epidemiológica de VIH/SIDA. Santiago: Ministerio de Salud; 2011.

41. Caro I, Guajardo G. Homofobia cultural en Santiago de Chile. Un estudio cualitativo. Santiago: Facultad Latinoamericana de Ciencias Sociales; 1997.

42. Sevilla ML, Álvarez N. Normalización del discurso homofóbico: aspectos bioéticos. Acta Bioeth 2006; 12:211-7.

43. Gates G, Ost J. Estimating the size of the gay and lesbian population. In: Gates G, Ost J, editors. The gay and lesbian atlas. Washington DC: The Urban Institute Press; 2004. p. 18-21.
44. Rostosky SS, Riggle EDB, Gray BE, Hatton RL. Minority stress experiences in committed same-sex couples relationships. Prof Psychol Res Pr 2007; 38:392-400.

45. Scherzer T. Negotiating health care: the experiences of young lesbian and bisexual women. Cult Health Sex 2000; 2:87-102.

46. Kertzner R, Meyer I, Frost D, Stirratt M. Social and psychological well-being in lesbians, gay men, and bisexuals: the effects of race, gender, age, and sexual identity. Am J Orthopsychiatry 2009; 79:500-10.

47. Riggle EDB, Whitman JS, Olson A, Rostosky SS, Strong S. The positive aspects of being a lesbian or gay man. Prof Pyschol Res Pr 2008; 39:210-7.

48. Domínguez-Fuentes JM, Hombrados-Mendieta MI, García-Leiva P. Social support and life satisfaction among gay men in Spain. J Homosex 2012; 59:241-55.

49. Rostosky SS, Korfhage B, Duhigg J, Stern A, Bennett L, Riggle EDB. Same-sex couples' perceptions of family support: a consensual qualitative study. Fam Process 2004; 43:43-56.

50. Schwartz S, Meyer IH. Mental health disparities research: the impact of within and between group analyses on tests of social stress hypotheses. Soc Sci Med 2010; 70:1111-8.

51. Eliason MJ, Schope RD. Does “don't ask don't tell” apply to health care? Lesbian, gay, and bisexual people's disclosure to health care providers. J Gay Lesbian Med Assoc 2001; 5:125-34.

52. Træen B, Martinussen M, Vitters J, Saini S. Sexual orientation and quality of life among university students from Cuba, Norway, India, and South Africa. J Homosex 2009; 56:655-69.

53. Doyle DM, Molix L. Perceived discrimination and wellbeing in gay men: the protective role of behavioural identification. Psychol Sex 2012; 1:1-14.

54. Mantecón A, Juan M, Calafat A, Becoña E, Román E. Respondent-driven sampling: un nuevo método de muestreo para el estudio de poblaciones visibles y ocultas. Adicciones 2008; 20:161-70.

55. Cárdenas M, Yañez S. Nuevas formas de muestreo para minorías y poblaciones ocultas: muestras por encuestado conducido en una población de inmigrantes sudamericanos. Univ Psychol 2012; 11:571-8.

56. Muhib FB, Lin LS, Stueve A, Miller RL, Ford WL, Johnson WD, et al. A venue-based method for sampling hard-to-reach populations. Public Health Rep 2001; 116:216-22.

57. Salganik M, Heckathorn D. Sampling and estimation in hidden population using respondet-driven sampling. Sociol Methodol 2004; 34:193-239.

Recibido el 29/May/2013

Versión final presentada el 03/Dic/2013

Aprobado el 12/Dic/2013 